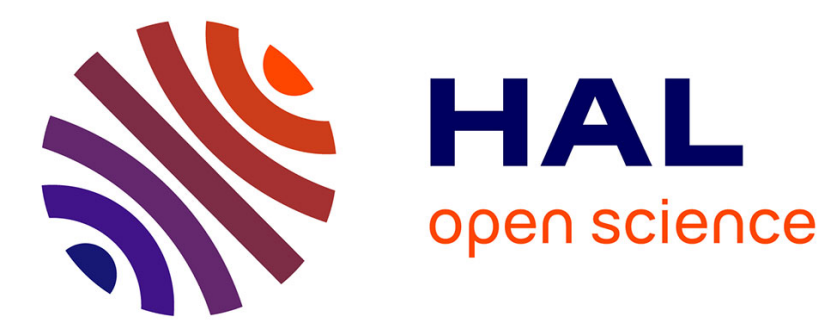

\title{
Statistical shape modeling of low level visual area borders.
}

\author{
Isabelle Corouge, Michel Dojat, Christian Barillot
}

\section{To cite this version:}

Isabelle Corouge, Michel Dojat, Christian Barillot. Statistical shape modeling of low level visual area borders.. Medical Image Analysis, 2004, 8 (3), pp.353-60. 10.1016/j.media.2004.06.023 . inserm00402298

\section{HAL Id: inserm-00402298 https://www.hal.inserm.fr/inserm-00402298}

Submitted on 1 Mar 2010

HAL is a multi-disciplinary open access archive for the deposit and dissemination of scientific research documents, whether they are published or not. The documents may come from teaching and research institutions in France or abroad, or from public or private research centers.
L'archive ouverte pluridisciplinaire HAL, est destinée au dépôt et à la diffusion de documents scientifiques de niveau recherche, publiés ou non, émanant des établissements d'enseignement et de recherche français ou étrangers, des laboratoires publics ou privés. 


\title{
Statistical shape modeling of low level visual area borders
}

\author{
Isabelle Corouge ${ }^{\mathrm{a}, *}$, Michel Dojat ${ }^{\mathrm{b}}$, Christian Barillot ${ }^{\mathrm{a}}$ \\ ${ }^{a}$ Visages Team, IRISAIINRIA-CNRS, Campus de Beaulieu, 35042 Rennes Cedex, France \\ ${ }^{\mathrm{b}}$ UM INSERM-UJF U594, LRC CEA 30V, CHU de Grenoble, Pavillon B, BP 317, 38043 Grenoble Cedex 09, France
}

Available online 7 August 2004

\begin{abstract}
This paper proposes a statistical modeling of functional landmarks delimiting low level visual areas which are highly variable across individuals. Low level visual areas are first precisely delineated by fMRI retinotopic mapping which provides detailed information about the correspondence between the visual field and its cortical representation. The model is then built by learning the variability within a given training set. It relies on an appropriate data representation and on the definition of an intrinsic coordinate system common to all visual maps. This allows to build a consistent training set on which a principal component analysis is eventually applied. Our approach constitutes a first step toward a functional landmark-based probabilistic atlas of low level visual areas. (C) 2004 Elsevier B.V. All rights reserved.
\end{abstract}

Keywords: Neuroimaging probabilistic atlas; Functional landmark; Visual areas; Retinotopy; Statistical modeling

\section{Introduction}

In the context of neuroimaging probabilistic atlases, this paper focuses on the human visual cortex and more precisely on the statistical modeling of functional landmarks delimiting low level visual areas. The number of areas in the visual cortex although estimated to be around 30 , as well as their exact role in the distributed processing of visual information, are not consensually determined yet. In this paper, we consider the following low order visual areas: V1, V2, V3, V3A and V4 of which a schematic $2 \mathrm{D}$ unfolded map can be found in Fig. 1. As the denomination of these visual areas is still under debate, we adopt the notation presented in (Zeki, 2003), where V4 is ventrally located along the ventral part of V3 in the inferior part of the occipital lobe. Note that "ventral", respectively "dorsal", qualifies areas lo-

\footnotetext{
${ }^{*}$ Corresponding author. Present address: Department of Computer Science, CB\#3175, Sitterson Hall, Chapel Hill, NC 27599-3175, USA Tel.: +1-919-966-7631; fax: +1-919966 4180 .

E-mail addresses: corouge@unc.edu (I. Corouge), Michel.Dojat@, ujf-grenoble.fr (M. Dojat), Christian.Barillot@irisa.fr (C. Barillot).
}

cated below, respectively above, the calcarine sulcus. We will use suffixes "d" and "v" to designate the dorsal and ventral parts of V1, V2 and V3. In this functional framework, the calcarine sulcus appears to be a significant anatomical landmark. Superior areas, i.e. in parietal zone, are dorsal areas and inferior areas, i.e. in temporal zone, are ventral areas. According to the visual stimulus, the visual information is more likely processed by the ventral or dorsal areas. It is widely but not unanimously admitted that spatial vision (motion and position) is mainly associated to dorsal areas whereas object vision (shape and color) is associated to ventral areas.

The low level visual areas benefit from the retinotopic property: a one-to-one correspondence exists between the retina and its representation on the cortical surface of each of these areas. This forms an homeomorphism between a part of the visual field and the cortical surface within a given area. In other words, a two adjacent points stimulation in the visual field leads to a two neighboring points activation in the cortical surface. Positions in the visual field are usually encoded by eccentricity and polar angle. A null eccentricity, which 


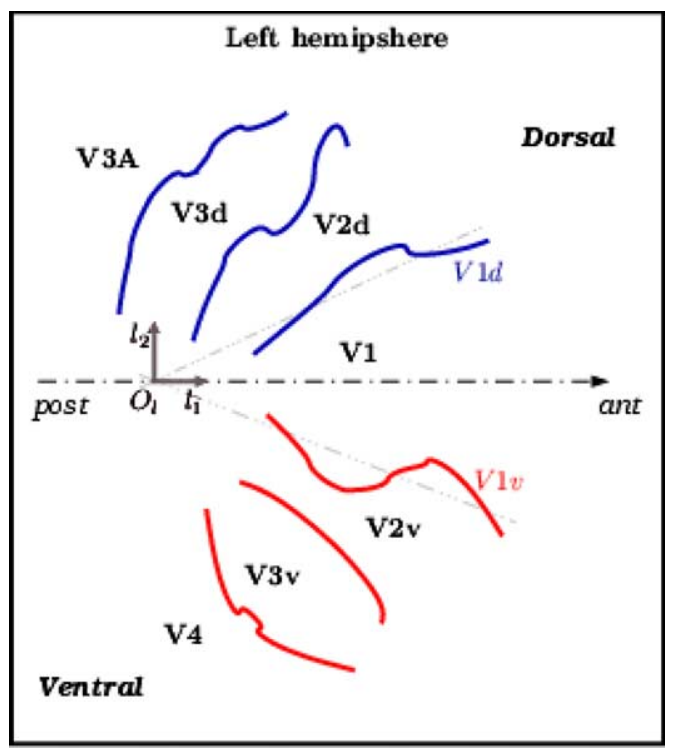

Fig. 1. Schematic representation for the left hemisphere of an unfolded map of the considered visual areas. The coordinate system intrinsic to such a map is superimposed.

corresponds to the center of gaze, is represented at the most posterior primary visual cortex part while representations of increasing eccentricities move toward more anterior parts of the cortical surface. Thus, the fovea, which is a tiny "stain" on the retina located very close to the optical axis and responsible for the central vision, is represented near the occipital pole. The upper quadrant of the controlateral hemifield is projected below the calcarine sulcus onto ventral areas whereas the lower quadrant is projected above the calcarine sulcus onto dorsal areas. V1, the primary visual area, receives information directly from the retina via the lateral geniculate nucleus and is located within the calcarine sulcus. The calcarine sulcus fundus is the cortical representation of the horizontal meridian. This latter also projects onto $\mathrm{V} 2 \mathrm{~d} / \mathrm{V} 3 \mathrm{~d}$ and $\mathrm{V} 2 \mathrm{v} / \mathrm{V} 3 \mathrm{v}$ borders. This alternates with the vertical meridian cortical representation located at $\mathrm{V} 1 / \mathrm{V} 2, \mathrm{~V} 3 \mathrm{~d} / \mathrm{V} 3 \mathrm{~A}$ and V3v/V4 borders. Thus, the representation of the controlateral hemifield is mirrored between adjacent visual areas.

Retinotopic properties can be exploited to precisely delineate low order visual areas by fMRI retinotopic mapping (Engel et al., 1994; Sereno et al., 1995; Warnking et al., 2002). Locations of these visual areas are highly variable across individuals (Amunts et al., 2000; Hasnain et al., 1998). Prior to any variability analysis, a spatial normalization has to be performed to express all subjects in a common frame. Various frameworks have been proposed. Van Essen et al. (2001) register individual data to a surface-based atlas by a surfacebased fluid warping technique constrained by geographical and/or functional landmarks. Most other approaches perform an inter-subject alignment based only on anatomical information. For instance, Hasnain et al. (1998) globally normalize each individual to the stereotaxic space, the Talairach atlas. Kochunov et al. (2003) propose a high resolution regional registration algorithm, using tissue classification, to remove anatomical differences between subjects by warping them into one reference brain. Besides, various features have been exploited for inter-individual variability analysis. For instance, Amunts et al. (2000) compare volume, extents and position of Brodmann's areas 17 and 18 while Hasnain et al. (1998) compute statistics on center of activations of each considered visual area (obtained by averaging the coordinates for its borders). In this paper, we propose a statistical modeling of the functional landmarks delimiting low level visual areas, i.e., of the low level visual area borders, in order to grasp the high inter-individual variability. We learn the variability within a given population and derive some statistics and shape occurrence probabilities from a statistical analysis, the principal component analysis (PCA). The training is based on a parametric representation of the structures of interest and on the definition of an intrinsic coordinate system for these structures in which instances of the training population can be matched. This model is entirely built upon functional landmarks rather than upon anatomical ones.

In the following section, we describe the visual area delineation process before building the statistical model in Section 3. Experiments and results are presented in Section 4. Finally, in Section 5, we discuss the proposed approach.

\section{Delineation of low level visual area borders}

The complete process to precisely delineate low level visual area borders is described in detail in (Warnking et al., 2002). We briefly recall the principle and the main steps of this process. It relies on retinotopic properties, periodic stimulation and exploits the orientation change of the representation of the local visual field between adjacent areas. It is performed by fMRI retinotopic mapping in three steps: measurements of functional data and 3D analysis, modeling of the cortical surface of interest and effective delineation.

\subsection{Measurements of functional data and $3 D$ analysis}

The visual stimuli used allow to determine the correspondence between a position in the visual field and its representation onto the cortical surface. Four periodic stimuli are considered: a dilating/contracting ring and clockwise/counter-clockwise rotating wedges as shown in Fig. 2. In low level areas, these stimulations induce a periodic travelling wave whose the phases are function of the position in the visual field. The corresponding 

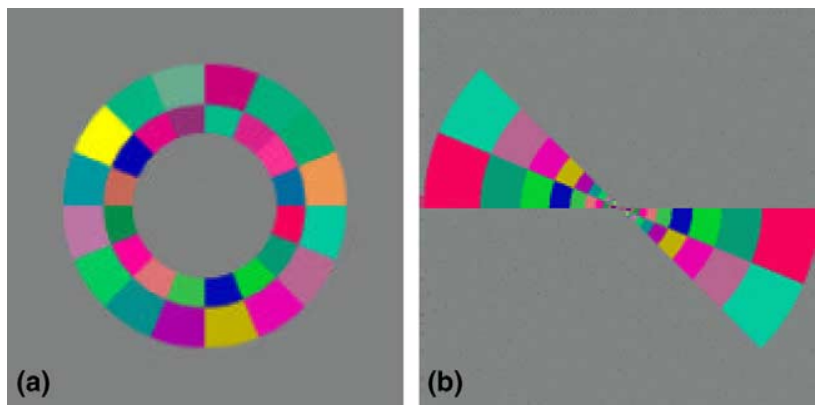

Fig. 2. Visual stimuli. (a) A dilating/contracting ring; (b) clockwise/ counter-clockwise rotating wedges.

periodic BOLD activation is recorded in $3 \mathrm{D}$, a Fourier analysis is performed and then phase-maps are obtained.

\subsection{Modeling of the cortical surface of interest}

In parallel, the center of the grey matter, which is the cortical surface of interest where functional activations are expected, is extracted from a tissue classification procedure followed by a dilatation of the grey matter/white matter interface. Since a bidimensional representation is preferable for visualization purposes, an unfolding algorithm (Guérin-Dugué et al., 2000) is applied to flatten the cortical surface, this one being restricted to the visual cortex area of interest. This algorithm enables to visualize the whole surface while resorting to no cuts and so preserves the original connectivity.

\subsection{Delineation of visual areas}

A surface representation of the cortical response can now be obtained. Voxels are projected on the unfolded cortex map and projections are interpolated by a Gaussian smoothing. A measure called "local visual field ratio" (VFR) is eventually computed to perform delineation (Sereno et al., 1994):

$\operatorname{VFR}=\frac{\partial\left(\phi_{r}, \phi_{\alpha}\right)}{\partial(u, v)}$

where $\left(\phi_{r}, \phi_{\alpha}\right)$ are the phases of observed responses corresponding to eccentricity $r$ and polar angle $\alpha$ of the visual field, the cortical surface being parameterized by $(u, v)$. The sign of VFR has a very precise interpretation: its change indicates a change in the orientation of the representation of the visual field. Therefore, visual area borders correspond to zero level of VFR. Besides, an uncertainty concerning the delineated lines can be estimated by the area having VFR values close to 0 .

Fig. 3 shows two examples of such delineated maps. The fMRI data used in these experiments has been acquired in PRESTO 3D sequences and their spatial resolution is $3 \times 3 \times 4 \mathrm{~mm}^{3}$. Three high resolution (about 1 $\mathrm{mm}^{3}$ ) anatomical MR data sets have been acquired per subject and averaged in order to increase contrast.
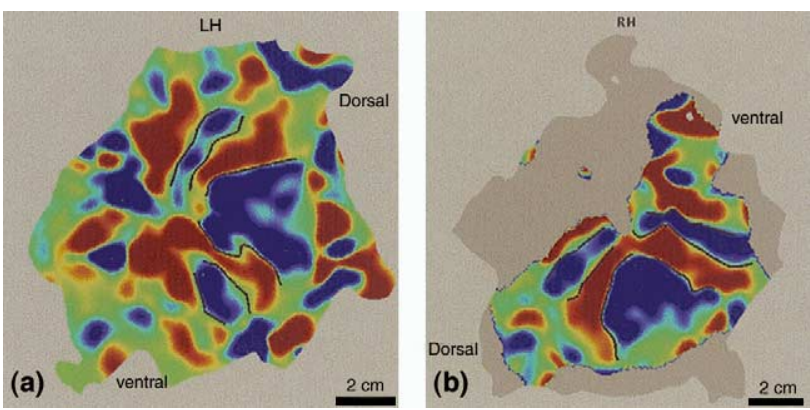

Fig. 3. Examples of unfolded retinotopic 2D maps showing VFR sign (color) and visual area borders (black lines) for the (a) left and (b) right hemispheres of one subject.

A good reproducibility of the delineation method has been demonstrated in (Warnking et al., 2002).

\section{Statistical modeling of low level visual area borders}

We propose now a statistical model of a delineated map, i.e., of the set of the six borders $V 1 d / V 2 d, V 1 v /$ $\mathrm{V} 2 \mathrm{v}, \mathrm{V} 2 \mathrm{~d} / \mathrm{V} 3 \mathrm{~d}, \mathrm{~V} 2 \mathrm{v} / \mathrm{V} 3 \mathrm{v}, \mathrm{V} 3 \mathrm{~d} / \mathrm{V} 3 \mathrm{~A}$ and $\mathrm{V} 3 \mathrm{v} / \mathrm{V} 4$. Our model is based on a training of the variability within a given population and is finally obtained by a wellknown multidimensional data analysis, the principal component analysis (PCA). The building of the training population first requires the definition of a data representation and second a matching scheme of these data. We assume relevant to establish a dense point to point correspondence between the set of maps and to derive theses correspondences from a parametric data representation once the population is aligned on a common coordinate system which has to be defined. In summary, building the training set consists in providing the data with a parametric representation, to sample this representation such that each of the six borders has the same number of sample points across the training set and to register this population on a common coordinate system in which lines will be matched point to point.

\subsection{Data parameterization and resampling}

The delineation method produces visual area borders of interest as 2D open lines described by a set of sampling points. On account of the natural variability as well as of the extraction process, these lines show variable shapes either more straight and broken, or more curved and smooth. Moreover, the initial sampling is irregular: sample points on the curve are not equidistant, some curve pieces are over-sampled like straight parts for example and interruptions can be observed. However, these latter ones are not functionally significant. We have chosen to use cubic B-spline curves to fit the initial data. This choice is indeed well adapted to model 
a wide range of different curves, to resample the data with the guaranty of a regular sampling, to interpolate missing data and so to handle interruptions.

Let $n b p$ be the initial number of sample points of a given border $c$. A set of $n b c$ control points is first generated, from which it is then possible to generate a new set of $n b p^{r}$ points which regularly sample the border $c$. For each line, the number of sampled points, $n b p_{l, l=1, \ldots, 6}^{r}$, is chosen such that each border is provided with a satisfactory representation across all the subjects. If borders are initially represented with a similar initial number of sampled points, then each border is oversampled on the most sampled instance. Otherwise, initially over-sampled borders are sub-sampled with $n b p^{r}$ performing a compromise of the number of sampled points of this border over the set of subjects. The choice of $n b p^{r}$ never leads to an effective sub-sampling of the initial border.

Each of the six borders of interest is thus represented by a set of $n b p_{l, l=1, \ldots, 6}^{r}$ points sampled on a cubic B-spline curve. Such a border can also be advantageously represented by the set of the $n b c_{l, l=1, \ldots, 6}^{r}$ control points associated with these new sampled points. This latter representation will be exploited in the statistical analysis step.

\subsection{Matching}

The alignment of the training set is based on the definition of an intrinsic coordinate system that we consider to be common to all instances in the training set. Let $\mathscr{R}_{l}\left(O_{l}, \mathbf{l}_{1}, \mathbf{l}_{2}\right)$ be this intrinsic coordinate system. $\mathscr{R}_{l}$ is determined for each instance in a geometric way only from V1 borders, i.e., from lines V1d/V2d and V1v/ $\mathrm{V} 2 \mathrm{v}$. V1 indeed appears as a central location to define an intrinsic coordinate system. Functionally, V1 plays a crucial role in primate vision. Most information first reaches $\mathrm{V} 1$ before spreading through the vast visual area network. Based on various techniques (Tootell et al., 1998), V1 is well anatomically defined; it is located within the calcarine sulcus, extending over its depth and lips. Moreover, with our functional retinotopic mapping technique, the fMRI signal to noise ratio is high in this area allowing a robust delineation of V1 borders.

The $x$-axis is defined as the bissectrice of the axes of inertia of $\mathrm{V} 1 \mathrm{~d} / \mathrm{V} 2 \mathrm{~d}$ and $\mathrm{V} 1 \mathrm{v} / \mathrm{V} 2 \mathrm{v}$ and follows the postero-anterior direction. It is functionally and anatomically significant. Functionally, this axis is close to the projection of the horizontal meridian, located in the middle of $\mathrm{V} 1$ at the frontier $\mathrm{V} 1 \mathrm{~d} / \mathrm{V} 1 \mathrm{v}$, and roughly separates dorsal and ventral areas. Anatomically, it is close to the calcarine sulcus fundus (this sulcus is not segmented yet from the available data) (Tootell et al., 1998; Wandell, 1995). Axis $y$ is defined orthogonal to $x$-axis and is directed towards dorsal, respectively ventral, areas for the left, respectively right, hemisphere.
The origin of $\mathscr{R}_{l}$ is defined as the intersection of the inertia axes of V1d/V2d and V1v/V2v. Fig. 1 illustrates a schematic unfolded map with the six delineated borders and the intrinsic coordinate system for the left hemisphere.

The training population is then aligned on $\mathscr{R}_{l}$ by a change of basis from the intrinsic coordinate system to the original coordinate system of each subject $\mathscr{R}\left(O, \mathbf{i}_{1}, \mathbf{i}_{2}\right)$. This can be expressed by the rigid transformation, i.e., a rotation and a translation, matrix $\mathbf{M}$. In homogeneous coordinates,

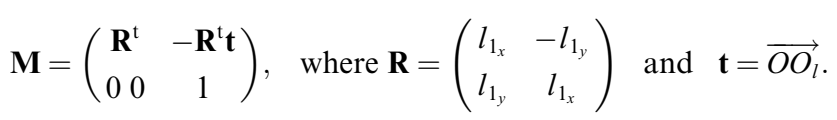

For each subject, this rigid transformation is computed and applied to every point of each line. Retinotopic maps of all subjects are aligned by rigid transformation on a coordinate system intrinsic to their structure. Once all the maps are expressed in $\mathscr{R}_{l}$, the matching consists in pairing control points having the same curvilinear abscissae. For instance, this matching scheme leads to appariate curves end points.

\subsection{Statistical analysis}

Let $\mathscr{P}$ be the training population made up of $N$ observations of maps $\mathbf{x}_{i, i=1, \ldots, N}$ resampled and registered as described above. Any map $\mathbf{x}$ is represented by the control points vector of the splines representing each of the six borders of interest:

$\mathbf{x}=\left(x_{0}^{1}, y_{0}^{1}, \ldots, x_{n b c_{1}^{r}-1}^{1}, y_{n b c_{1}^{r}-1}^{1}, \ldots, x_{0}^{6}, y_{0}^{6}, \ldots, x_{n b c_{6}^{r}-1}^{6}, y_{n b c_{6}^{r}-1}^{6}\right)^{\mathrm{t}}$,

where $\left(x_{i}^{l}, y_{i}^{l}\right)$ are the $n b c_{l}^{r}$ control points of the $l$ th line of the map $(l=1, \ldots, 6)$.

We perform on $\mathscr{P}$ a principal component analysis. This analysis characterizes the variations within a given training population and extracts the principal modes of deformation relative to the mean map. Briefly, PCA consists in expressing the observations in a new basis, also orthogonal, with mean map $\overline{\mathbf{x}}$ as an origin and eigenvectors or modes of the observations covariance matrix $\mathbf{C}$ as axes. An approximation of these observations can be obtained by truncating a certain number of modes. The reconstructed observation is then written as $\mathbf{x}=\overline{\mathbf{x}}+\Phi_{m} \mathbf{b}_{m}$, where $m$ is the number of retained modes, $\Phi_{m}$ the truncated modes matrix and $\mathbf{b}_{m}=\left(b_{i}\right)_{i=1, \ldots, m}$ the $m$-dimensional vector representing the original observation in the truncated modal basis. Reconstruction quality can be measured by

$\tau=\sum_{i=1}^{m} \lambda_{i} / \lambda_{\mathrm{T}}$ 
where $\lambda_{i}$ is the $i$ th eigenvalue, in decreasing order, of matrix $\mathbf{C}$ and corresponds to the variance explained by the $i$ th mode, and where $\lambda_{\mathrm{T}}$ is the total variance.

Under the assumption that the distribution of the elements of $\mathscr{P}$ is Gaussian, the variation of $b_{i, i}=1, \ldots, m$ in an interval such as $\left[-3 \sqrt{\lambda_{i}}, 3 \sqrt{\lambda_{i}}\right]$ provides an indication of the variability of the studied object class. This indication is all the more reliable and realistic as the training set contains a sufficient number of representative instances.

\section{Experiments and results}

Experiments have been led on an 8 subjects database made up of 4 females and 4 males, $36 \pm 10$ years old, with normal vision. Since the extraction of the V3v/V4 right border has failed for one subject, only 7 subjects have been used for the right hemisphere.

\subsection{Data parameterization and resampling}

Each border has been parameterized and resampled as described in Section 3.1. The number $n b c$ of control points computed from the $n b p$ original sampled points has been fixed empirically such that $n b c=n b p / 2$. In theory, data representation is all the more fine as the ratio $n b c / n b p$ is close to 1 . In practise, due to the non-uniformity of the initial curve sampling, a ratio equal to 2 has appeared sufficient. Fig. 4 shows some examples of resampled curves with initial curves superimposed. It indicates a very good conservation of the global original shape and of its position. In order to quantify the error induced by resampling, we have computed the Hausdorff distance between resampled lines and initial lines, for each border and each subject. Given two sets of points $S_{1}$ and $S_{2}$, the Hausdorff distance $H$ is defined as

$$
\begin{aligned}
& H\left(S_{1}, S_{2}\right)=\max \left(h\left(S_{1}, S_{2}\right), h\left(S_{2}, S_{1}\right)\right), \\
& \quad \text { with } h\left(S_{1}, S_{2}\right)=\max _{s_{1} \in S_{1}} \min _{S_{2} \in S_{2}}\left\|s_{1}-s_{2}\right\|,
\end{aligned}
$$

$h\left(S_{1}, S_{2}\right)$ is the directed Hausdorff distance from $S_{1}$ to $S_{2}$, $\|\cdot\|$ being the Euclidean norm. The Hausdorff distance measures the distance from the point in $S_{1}$ the furthest to the point set $S_{2}$ and vice versa. Hence, it is sensitive to a large error and provides somehow the maximal error resampling. The average of this distance over the set of subjects as well as its standard deviation are presented
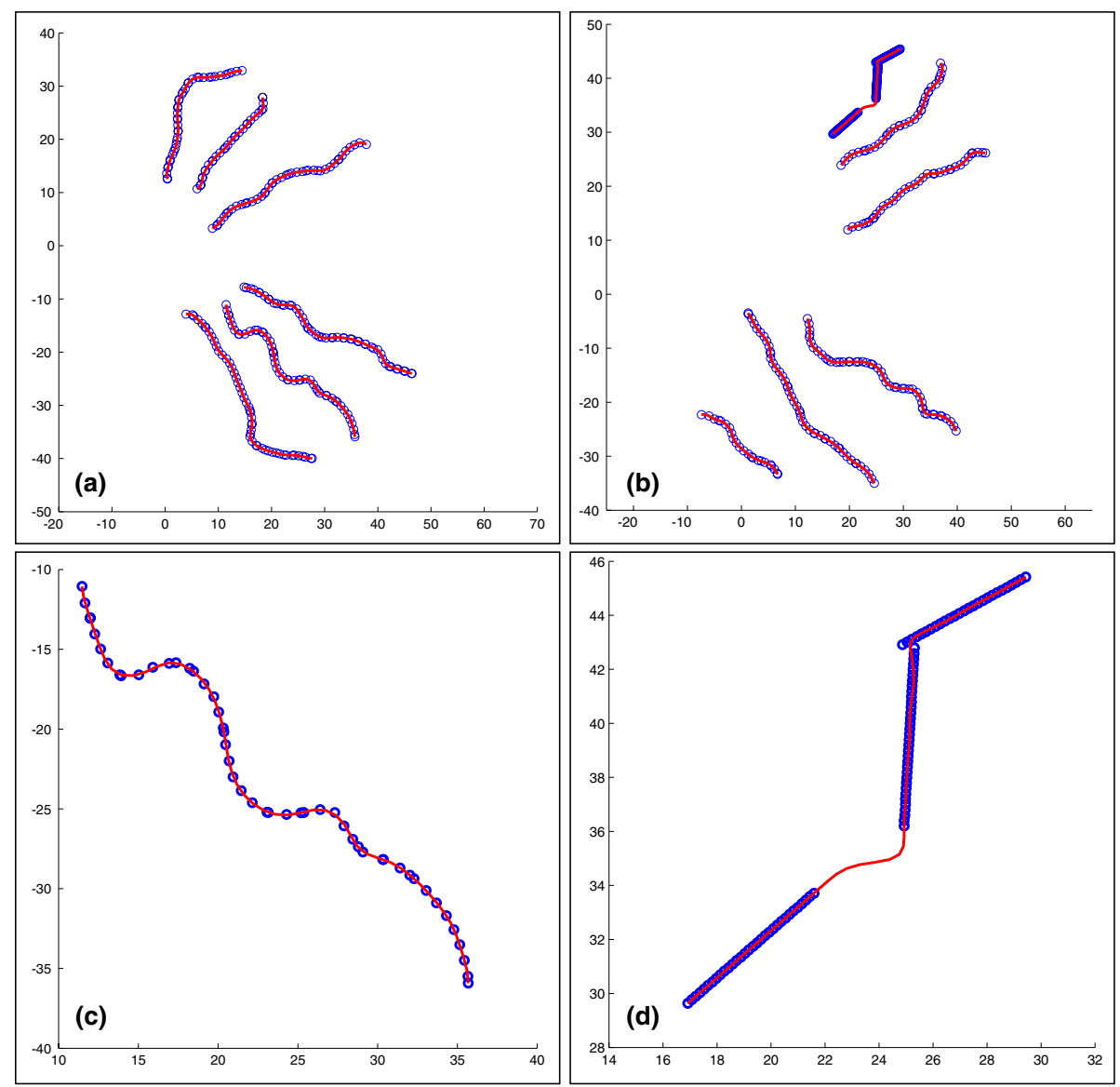

Fig. 4. Resampling. (a) and (b) Examples of individual resampled maps for (a) left hemipshere and (b) right hemisphere. Blue dots are the initial sampled points while red lines are resampled borders. (c) and (d) Zoom on two individual particular borders: V1v/V2v in the left hemisphere and $\mathrm{V} 3 \mathrm{v} / \mathrm{V} 4$ in the right hemisphere. 

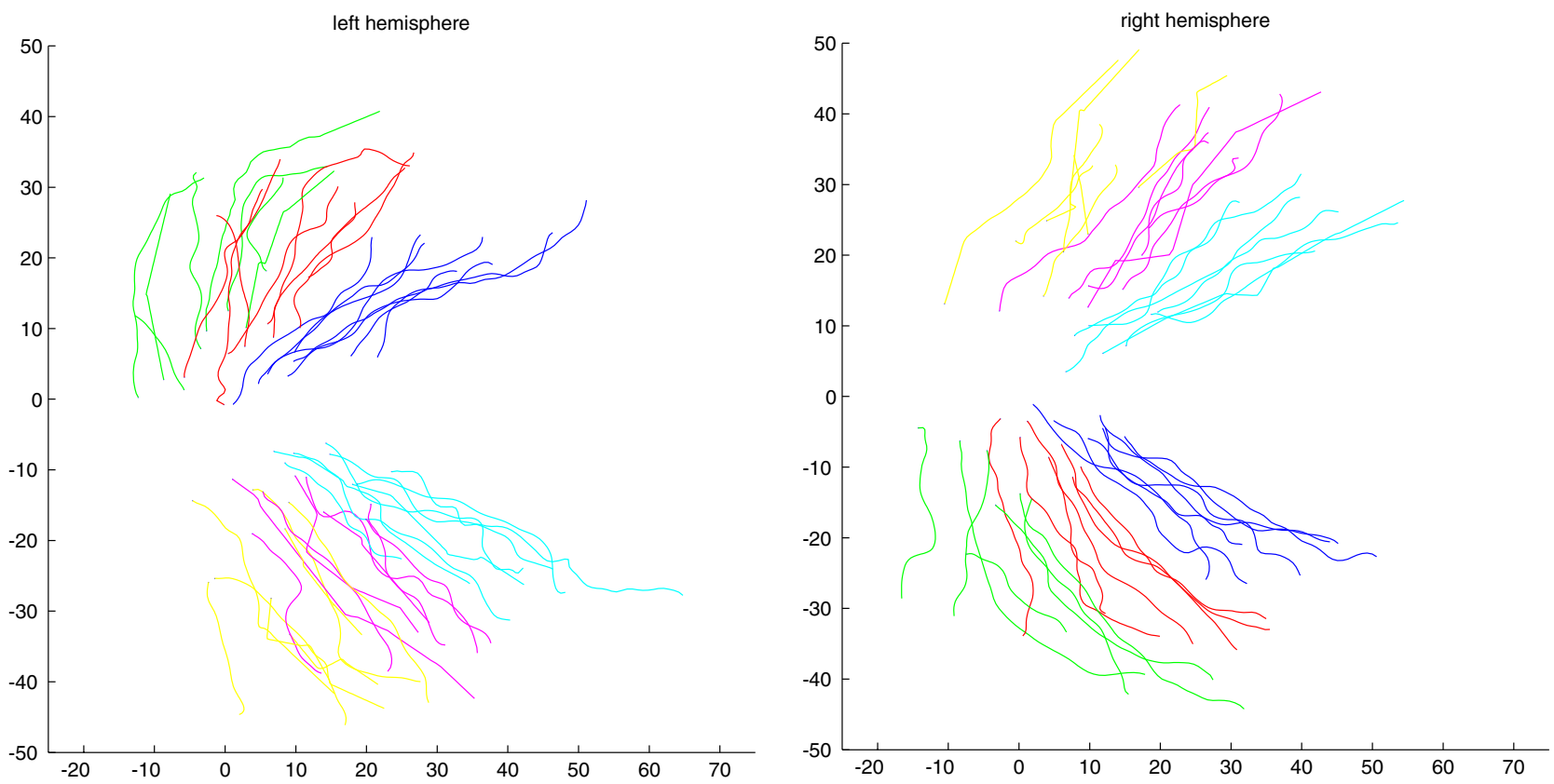

Fig. 5. Training populations corresponding to left and right hemispheres. They are locally registered in the intrinsic coordinate system. V1d/V2d is represented in blue, V1v/V2v in cyan, $\mathrm{V} 2 \mathrm{~d} / \mathrm{V} 3 \mathrm{~d}$ in red, $\mathrm{V} 2 \mathrm{v} / \mathrm{V} 3 \mathrm{v}$ in magenta, V3d/V3A in green and V3v/V4 in yellow.

in Table 1. The error is weak and stable over the database.

\subsection{Alignment}

Fig. 5 shows the borders locally registered onto the intrinsic coordinate system for both hemispheres. We can observe a relatively important variability within these populations, in particular as the position is concerned. This variability increases when moving away from V1. Note that the precision on the phase measurement, at the basis of the delineation, is directly linked to the signal to noise ratio (SNR), which decreases when moving away from V1.
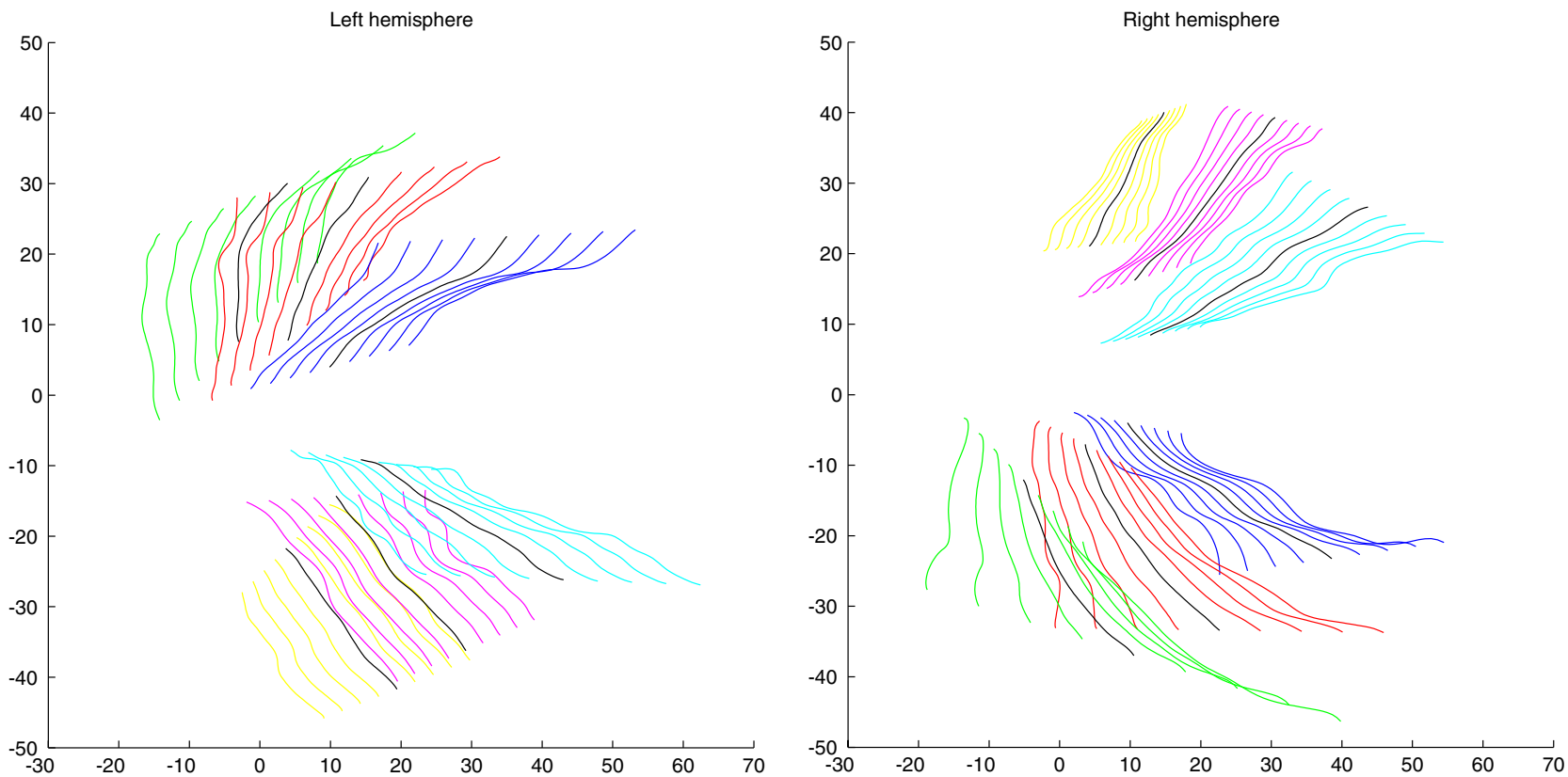

Fig. 6. For both hemispheres, variations according to the first mode around the mean map (black lines): $-2 \sqrt{\lambda_{1}} \leqslant b_{1} \leqslant+2 \sqrt{\lambda_{1}}$. V1d/V2d is represented in blue, $\mathrm{V} 1 \mathrm{v} / \mathrm{V} 2 \mathrm{v}$ in cyan, $\mathrm{V} 2 \mathrm{~d} / \mathrm{V} 3 \mathrm{~d}$ in red, $\mathrm{V} 2 \mathrm{v} / \mathrm{V} 3 \mathrm{v}$ in magenta, $\mathrm{V} 3 \mathrm{~d} / \mathrm{V} 3 \mathrm{~A}$ in green and $\mathrm{V} 3 \mathrm{v} / \mathrm{V} 4$ in yellow. 
Table 1

For each border and each hemisphere, average and standard deviation in $\mathrm{mm}$ over all the subjects of the Hausdorff distance between initial lines and resampled lines

\begin{tabular}{lll}
\hline Borders & Left hemisphere & Right hemisphere \\
\hline V1d/V2d & $0.562 \pm 0.009$ & $0.561 \pm 0.004$ \\
V1v/V2v & $0.603 \pm 0.010$ & $0.770 \pm 0.073$ \\
V2d/V3d & $0.620 \pm 0.019$ & $0.571 \pm 0.005$ \\
V2v/V3v & $0.612 \pm 0.026$ & $0.573 \pm 0.019$ \\
V3d/V3A & $0.480 \pm 0.020$ & $0.586 \pm 0.011$ \\
V3v/V4 & $0.522 \pm 0.016$ & $0.587 \pm 0.100$ \\
\hline
\end{tabular}

Table 2

Percentage, $\tau_{p}$, of cumulative variance according to the number of modes retained for left and right hemisphere; $\tau_{p}=\sum_{i=1}^{m} \lambda_{i} / \lambda_{\mathrm{T}} \times 100$

\begin{tabular}{lcc}
\hline Modes & Left hemisphere & Right hemisphere \\
\hline 1 & 70.2 & 60.0 \\
2 & 84.1 & 82.6 \\
3 & 89.8 & 90.0 \\
4 & 94.2 & 95.1 \\
5 & 96.7 & 98.6 \\
6 & 98.8 & 100 \\
7 & 100 & 100 \\
\hline
\end{tabular}

\subsection{Statistical analysis}

The statistical analysis has been applied on the control points of the splines defining the borders previously resampled and registered. The number of control points, $n b c_{l}^{r}$, has been fixed such that $n b c_{l}^{r} / n b p_{l}^{r}=4$. This choice induces an observation vector with 90 points and so 180 variables. It produces a fine representation of the lines to model while avoiding a huge dimensionality in regards to the cardinal of the training population. Table 2 exhibits the percentage of cumulative variance for each hemisphere according to the number of modes retained.

The first mode strongly predominates. Fig. 6 shows the variations around the mean map along the first mode. We observe a border variability both in shape and position. However, position variability appears as the most important. We also note that borders appear less variable on the ventral side of the right hemisphere. These results, although interesting, should be interpreted in regards to the cardinal of the training population. In this restricted experimental framework, their scope is limited.

\section{Discussion and perspectives}

We have proposed an approach to statistically model functional borders delimiting low order visual areas. The parameterization we proposed is adapted to the data to be treated. However, further experiments could help to determine optimal parameters in particular concerning the number of sampled points to use in the resampling stage and the number of control points to use in the statistical analysis stage. The definition of the intrinsic coordinate system has been constrained by presently available information. It could benefit from more anatomical and functional information. In particular, it would be interesting to effectively use the calcarine sulcus as a landmark. The calcarine's segmentation is far from being a trivial task and is currently work in progress. The origin, determined upon a geometric criterion, could also benefit from more functional information. For instance, it could be extracted from an area (measurements are marred with uncertainty and depend on SNR) responding to some predefined eccentricity and polarity. The use of the fovea representation on the cortical surface could also be investigated. Although this representation is not punctual, it could serve as a basis to define the origin. The matching process depends on the extraction technique, on the parameterization and on the definition of the intrinsic coordinate system. If the extraction partially failed such that some borders are more or less truncated, this matching is not adequate anymore and introduces a bias in the model. In order to handle non-systematic missing data, two ways could be explored: improving the robustness of the extraction or using a baseddistance matching, nevertheless relevance of this latter cannot be systematically guaranteed. As mentioned before, our experimental framework is quite limited and an enlargement of our data set is desirable for a complete validation of such a modeling. It is largely demonstrated that functional borders and anatomical structures such as sulci are highly variable among individuals namely in function of age and sex. For instance, the location, size and shape of V1 and of the calcarine sulcus are significantly variable between subjects. Accordingly, the results we report on a small sample size (8 subjects) should not be directly generalized to a larger population. More experimental investigations are required for this purpose. Besides, our experimentation was concerned with retinotopic maps obtained at 1.5 $\mathrm{T}$. The use of an higher magnetic field, typically $3 \mathrm{~T}$, will allow us to improve the precision of the phase maps and thus the quality of our model.

In spite of the above limitations, the proposed method opens perspectives of real interest. It can be used in a given population (1) to study retinotopic area variations, (2) to realign functional scans based on functional visual landmarks or even on anatomical ones, e.g. calcarine sulcus, (3) to map inter-subject functional data onto the mean subject (Corouge et al., 2003) and finally (4) to build a functional and anatomical probabilistic atlas of retinotopic areas for a given population (Van Essen et al., 2001). Such an atlas could namely be exploited in mapping subjects for which functional 
recordings are not available but anatomical landmarks, e.g. calcarine sulcus, are.

\section{References}

Amunts, K., Malikovic, A., Mohlberg, H., Schormann, T., Zilles, K., 2000. Bordmann's areas 17 and 18 brought into stereotaxic spaceWhere and how variable?. NeuroImage 11, 66-84.

Corouge, I., Hellier, P., Gibaud, B., Barillot, C., 2003. Inter-individual functional mapping: a non linear local approach. NeuroImage 19 (4), 1337-1348.

Engel, S.A., Rumelhart, D.E., Wandell, B.A., Lee, A.T., Glover, G.H., Chichilnisky, E.J., Shadlen, M.N., 1994. fMRI of human visual cortex. Nature 369 (6481), 525.

Guérin-Dugué, A., Olympieff, S., Gisert-Lopez, J., Chéhikian, A., Warnking, J., Rubin, C., Segebarth, C. 2000. Représentation plane du cortex visuel en imagerie fonctionnelle à résonance magnétique. In: Proceedings of Reconnaissance des Formes et Intelligence Artificielle 2, pp. 29-38.

Hasnain, M.K., Fox, P.T., Woldorff, M.G., 1998. Intersubject variability of functional areas in the human visual cortex. Human Brain Mapping 6, 301-315.
Kochunov, P., Hasnain, M., Lancaster, J., Grabowski, T., Fox, P., 2003. Improvement in variability of the horizontal meridian of the primary visual area following high-resolution spatial normalization. Human Brain Mapping 18, 123-134.

Sereno, M.I., McDonald, C.T., Allman, J.M., 1994. Analysis of retinotopic maps in extrastriate cortex. Cerebral Cortex 4, 601-620.

Sereno, M.I., Dale, A.M., Reppas, J.B., Kwong, K.K., Belliveau, J.W., Brady, T.J., Rosen, B.R., Tootell, R.B.H., 1995. Borders of multiple visual areas in human revealed by functional magnetic resonance imaging. Science 268, 889-893.

Tootell, R.B.H., Hadjikani, N.K., Vanduffel, W., Liu, A.K., Mendola, J.D., Sereno, M.I., Dale, A.M., 1998. Functional analysis of primary visual cortex (V1) in humans. Proceedings of the National Academy of Sciences of the United States of America 95, 811-817.

Van Essen, D.C., Lewis, J.W., Drury, H.A., Hadjikhani, N., Tootell, R.B.H., Bakircioglu, M., Miller, M.I., 2001. Mapping visual cortex in monkeys and humans using surface-based atlases. Vision Research 41, 1359-1378.

Wandell, B.A., 1995. Foundations of Vision. Sinauer Associates, pp. $160-161$

Warnking, J., Dojat, M., Guérin-Dugué, A., Delon-Martin, C., Olympieff, S., Richard, N., Chéhikian, A., Segebarth, C., 2002. fMRI retinotopic mapping - step by step. NeuroImage 17 (4), 1665-1685.

Zeki, S., 2003. Improbable areas in the visual brain. Trends in Neurosciences 26 (1), 23-26. 